\title{
APLICAÇÃO DO MÉTODO DOS ELEMENTOS DISCRETOS NA AVALIAÇÃO DE CHUTES DE ESCOAMENTO DE MINÉRIOS DE FERRO ITABIRÍTICOS
}

\author{
M. E. ASSUMPÇÃO, D. B. MAZZINGHY*, L. D. SOUZA, J. F. C. RUSSO, B. A. BAILONA e N. SPOGIS \\ Anglo American e ESSS \\ douglas.mazzinghy@angloamerican.com*
}

Artigo submetido em novembro/2015 e aceito em dezembro/2015

DOI: $10.15628 /$ holos.2015.3666

\section{RESUMO}

Chutes de transferência de material podem ser pontos de gargalo de qualquer operação se não forem corretamente projetados. Recentemente, o modelamento de elementos discretos tem sido utilizado com sucesso na indústria mineral para simulação de processos envolvendo, principalmente, o manuseio de materiais. As vantagens do uso desta tecnologia são imensas visto que é possível visualizar em detalhe o escoamento de materiais em chutes e silos antecipando possíveis problemas de fluxo de material, ainda na fase de projeto. O presente trabalho tem como objetivo apresentar os resultados de diversas simulações conduzidas para avaliar o comportamento de um minério de ferro itabirítico através de um chute de bypass de um circuito de cominuição com prensas de rolos.

PALAVRAS-CHAVE: Chutes, Simulação, Método de elementos discretos, Itabiritos.

\section{APPLICATION OF THE DISCRETE ELEMENT METHOD TO EVALUATE A TRANSFER CHUTE FOR AN ITABIRITE IRON ORE}

\begin{abstract}
Material transfer chutes can be bottleneck points of any operation if not designed properly. Recently, the discrete element modeling has been successfully used in the minerals industry for simulating processes involving mainly material handling. The advantages of using this technology are immense as you can see in detail the flow of materials in chutes and silos anticipating possible
\end{abstract}

material flow problems, still in the design phase. This paper aims to present the results of several simulations conducted to evaluate the behavior of an itabirite iron ore through a bypass chute of a HPGR comminution circuit.

KEYWORDS: Chutes, Simulation, Discrete element modeling, Itabirite. 


\section{INTRODUÇÃO}

O circuito de cominuição do projeto Minas-Rio é composto por britagem primária com britadores de mandíbulas e grelhas vibratórias e por britagem secundária com britadores cônicos e peneiras vibratórias em circuito fechado. $O$ minério processado nestas etapas é direcionado para uma pilha pulmão. O minério retomado da pilha alimenta a etapa de moagem com prensas de rolos em circuito aberto e com moinho de bolas e hidrociclones em circuito fechado. Existe a possibilidade de bypass das prensas de rolos se o minério alimentado estiver com distribuição granulométrica mais fina e adequada para alimentação dos moinhos de bolas.

O projeto Minas-Rio iniciou as suas operações no final de 2014 e encontra-se na fase de ramp up. Durante o início das operações o material alimentado na planta estava com granulometria mais fina que a especificada para alimentação das prensas de rolos e então se decidiu fazer um bypass das prensas de rolos. $O$ chute de bypass das prensas de rolos apresentou problemas de escoamento do minério.

\subsection{Descrição do Método dos Elementos Discretos}

A ciência e tecnologia de partículas é uma área de pesquisa interdisciplinar em rápido desenvolvimento, sendo o seu núcleo o entendimento das relações entre propriedades micro e macroscópicas de material particulado ou granular - um estado da matéria amplamente encontrado, porém pouco compreendido. O comportamento macroscópico de um material particulado é controlado pelas interações entre partículas individuais, assim como interações com um gás ou líquido circundante e paredes. O entendimento dos mecanismos microscópicos em termos destas forças de interações é, portanto, a chave para a pesquisa interdisciplinar em materiais particulados, produzindo resultados que podem ser amplamente usados.

Diversas técnicas de modelagem discretas foram desenvolvidas, incluindo método de Monte Carlo, célula autônoma e método dos elementos discretos (DEM). As simulações com DEM podem fornecer informações dinâmicas, tais como trajetórias e forças transientes agindo em partículas individuais. Dois tipos de DEM são mais comuns: abordagem de partícula macia e partícula rígida. O método de abordagem de partícula macia originalmente desenvolvido por Cundall e Strack (1979), foi a primeira técnica de simulação de dinâmica de partícula publicado na literatura aberta. Em tal abordagem, é permitido que as partículas sofram deformações pequenas, e estas deformações são usadas para estimar forças elásticas, plásticas e de atrito entre as partículas. O movimento das partículas é descrito pelas bem estabelecidas leis do movimento de Newton. Uma característica dos modelos de partícula macia é que eles são capazes de lidar com múltiplos contatos entre partículas, o que é importante quando se modela sistemas quase estáticos. Em contrapartida, em uma simulação de partículas rígidas, uma sequência de colisões é processada, uma colisão de cada vez e sendo instantânea; frequentemente as forças entre partículas não são explicitamente consideradas. Portanto, tipicamente, o método de partículas rígidas é útil em escoamentos granulares rápidos.

Um modelo DEM bem calibrado pode ser utilizado para obter maiores conhecimentos sobre processos industriais. Alguns exemplos incluem estudos de escoamentos de materiais em chutes, silos e hoopers; cominuição de material em moinhos e britadores, cálculo de desgaste em 
equipamentos, entre outros. A força total experimentada por cada uma das partículas em um sistema granular é modelada e as subsequentes acelerações, velocidades e posições são controlados ao longo de um período de tempo. A força total é o somatório das forças de contato (partícula/partícula e partícula/ geometria), e as forças de corpo, como a gravidade, arrasto, campos magnéticos, ou forças eletrostáticas. A principal distinção entre DEM e dinâmica molecular $(M D)$ é que no DEM, as colisões de partículas finitas e rotações desempenham um papel dominante.

Uma partícula em um meio granular pode ter dois tipos de movimento: translacional e rotacional. Durante sua movimentação, a partícula pode interagir com suas partículas vizinhas ou paredes e interagir com o fluido ao seu redor, com o qual quantidade de movimento e energia são trocados. Estritamente falando, este movimento não é afetado somente pelas forças e torques originados das partículas e fluido da vizinhança imediata, mas também pelo fluido e partículas distantes pela propagação de ondas de perturbação. A complexidade de tais processos tem barrado qualquer tentativa de modelar este problema analiticamente. $\mathrm{Na}$ abordagem de DEM, geralmente é assumido que este problema pode ser resolvido escolhendo um passo de tempo menor do que um valor crítico tal que durante um único passo de tempo a perturbação da partícula e do fluído não pode propagar além das partículas e fluido vizinhos (Cundall e Strack, 1979). Desta forma, em todos os passos de tempo as forças resultantes em uma partícula podem ser determinadas exclusivamente de sua interação com as partículas em contato e o fluido ao seu redor em um sistema de partículas grosseiras. Para um sistema de partículas finas, forças que não são de contato como Van Der Waals e forças eletrostáticas deveriam ser incluídas. Baseada nestas considerações, a segunda lei de Newton do movimento pode ser usada para descrever o movimento de partículas individuais.

\subsection{Objetivo}

O presente trabalho tem como objetivo apresentar os resultados de diversas simulações conduzidas para avaliar o comportamento de um minério de ferro itabirítico através de um chute de bypass de um circuito de cominuição com prensas de rolos utilizando o método dos elementos discretos. As simulações foram realizadas no programa ROCKY que é um poderoso programa de DEM, que simula com rapidez e precisão o comportamento do fluxo granular de partículas de diferentes formas e tamanho dentro de equipamentos como correias transportadoras, chutes, moinhos, entre outros.

\section{MATERIAIS E MÉTODOS}

Uma amostra da alimentação da prensa de rolos foi coletada na planta para caracterização. Foram determinadas as densidades real e aparente, o ângulo de repouso, a umidade e a distribuição granulométrica. O formato das partículas foi selecionado segundo as opções disponíveis no ROCKY, conforme mostrado na Figura 1. 


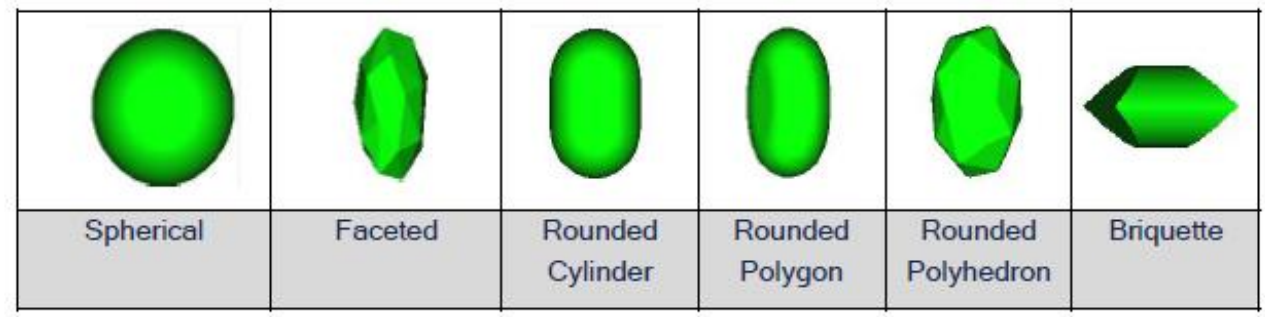

Figura 1: Formato de partículas disponíveis no Rocky

O sistema de moagem por prensa de rolos é apresentado na Figura 2.

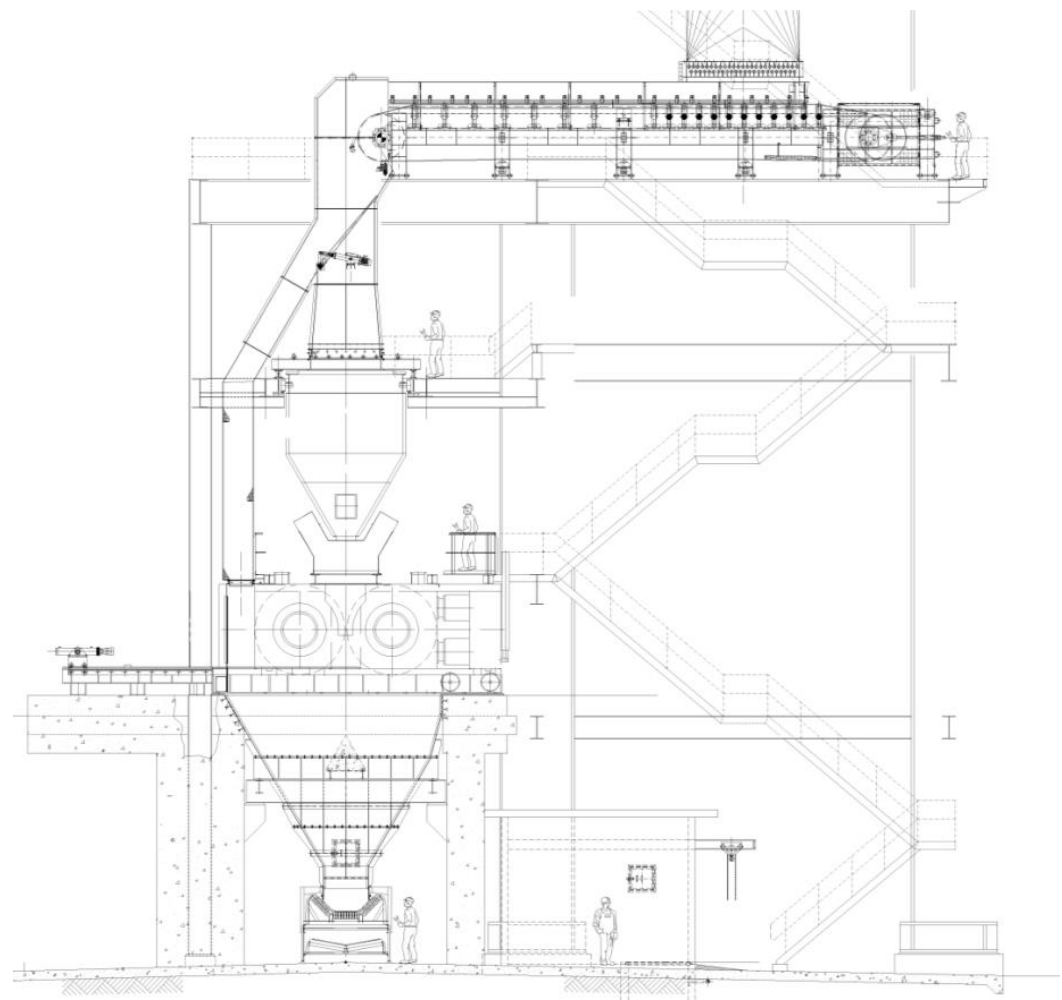

Figura 2: Vista lateral do sistema de moagem por prensa de rolos do Minas-Rio.

Um alimentador de correia direciona o minério a uma velocidade de $0,5 \mathrm{~m} / \mathrm{s}$ e a uma taxa de $3500 \mathrm{t} / \mathrm{h}$ no chute de alimentação das prensas de rolos. O chute de alimentação apresenta duas possibilidades de fluxo. O fluxo pode ser direcionado para alimentar a prensa de rolos ou pode ser feito um bypass. $O$ sistema de bypass é utilizado para condições adversas quando: não-britáveis forem detectados no sistema, granulometria mais fina que o especificado para moagem pela prensa de rolos ou intervenções para a manutenção. O desviador é acionado mecanicamente por sistema sem-fim sendo o responsável pelo direcionamento do fluxo no chute.

O caminho percorrido pelo fluxo no bypass depois de encontrar o desviador é apresentado a seguir. O minério escorre por uma rampa de 60 de inclinação até chegar em uma estrutura vertical que é composta por caixas de rochas. No final do chute de by-pass o minério é descarregado a cima e na traseira do rolo móvel. Este trajeto descrito compõem todo sistema de bypass. 
O modelo de elementos discretos utilizado neste estudo é apresentado na Figura 3. 0 modelo não contemplou o chute de alimentação da prensa de rolos. Apenas metade do rolo móvel foi considerada em sua posição de máxima pressão para verificação de impacto em sua estrutura.

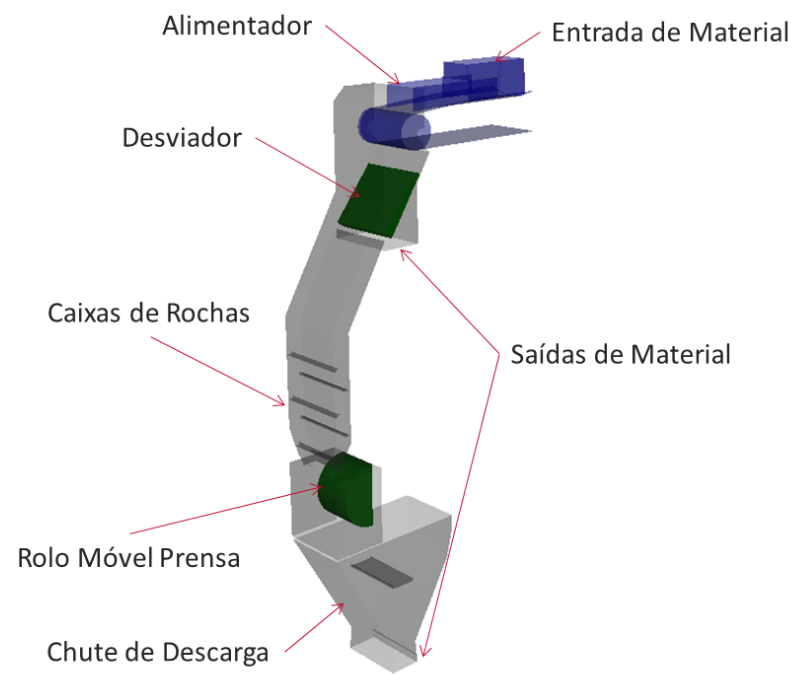

Figura 3: Modelo de elementos discreto do chute de by-pass das prensas de rolos.

A Tabela 1 apresenta os valores dos parâmetros utilizados na simulação de elementos discretos. Os valores do coeficiente de restituição e atrito estático foram obtidos através do estudo de Nasato (2011). Os valores de atrito estático e dinâmico foram considerados iguais devido à pequena variação.

Tabela 1: Valores dos parâmetros utilizados no software Rocky

\begin{tabular}{cccc}
\hline Variáveis & Minério/Minério & Minério/Borracha & Minério/Aço \\
\hline Coef. Restituição & 0,4 & 0,3 & 0,3 \\
Atrito estático & 0,65 & 0,6 & 0,5 \\
Atrito dinâmico & 0,65 & 0,6 & 0,5 \\
Distância adesiva & $5 \mathrm{~mm}$ & $5 \mathrm{~mm}$ & $5 \mathrm{~mm}$ \\
\hline
\end{tabular}

\section{RESULTADOS E DISCUSSÃO}

A amostra apresentou formato de partículas lamelares. Assim, foi selecionado no ROCKY o formato chamado "Faceted", por ser o que melhor representa a amostra estudada. A Tabela 2 apresenta os resultados da caracterização tecnológica da amostra.

Tabela 2. Caracterização tecnológica

\begin{tabular}{cc}
\hline Propriedades & Valores \\
\hline Densidade Real $\left(\mathrm{t} / \mathrm{m}^{3}\right)$ & 4,29 \\
Densidade Aparente $\left(\mathrm{t} / \mathrm{m}^{3}\right)$ & 2,52 \\
Ângulo de Repouso $\left({ }^{\circ}\right)$ & 40,5 \\
Umidade $(\%)$ & 10,0 \\
\hline
\end{tabular}


A Figura 4 apresenta a distribuição granulométrica da amostra.

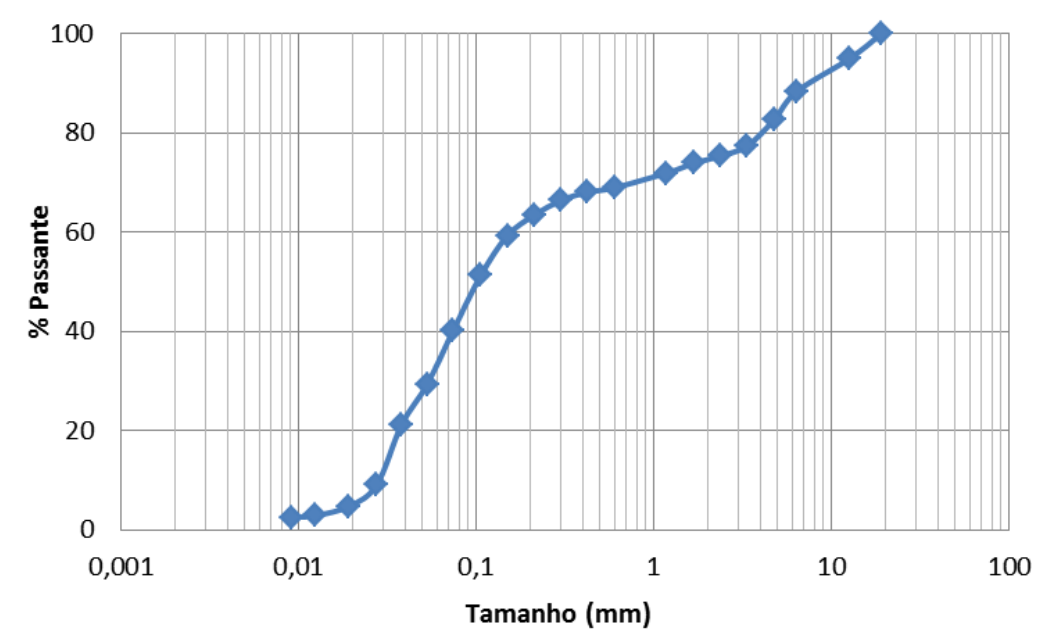

Figura 4: Distribuição granulométrica da amostra estudada.

A distribuição granulométrica da amostra estudada apresentou um formato bimodal com reduzida quantidade de grossos e elevada quantidade de finos, um comportamento característico de alguns minérios de ferro. Pode-se observar que mais de $70 \%$ das partículas são menores do que $1 \mathrm{~mm}$. A partir destes resultados foram inseridos na simulação os valores da densidade real e foi definido o valor de tamanho de partícula. O tempo total simulado foi de $40 \mathrm{~s}$. Com os resultados da simulação foram avaliados a velocidade das partículas, o impacto com as estruturas e o acumulo de material. O tempo de estabilização da simulação foi de $18 \mathrm{~s}$.

Observou-se na simulação uma alta incidência de impacto no modulo de transição da rampa para a estrutura vertical, conforme apresentado na Figura $5(A)$. Esta região de alto impacto pode ser explicada devido a alta velocidade do fluxo aliada a uma mudança abrupta de direção. Foi observado em regime de operação o desgaste prematuro desta região necessitando o seu reparo com placas de revestimento. A Figura 5 (B) mostra o local de instalação das placas de desgaste. Os desenhos do chute foram revisados e esta região recebeu placas de revestimento.

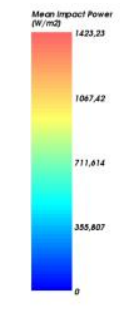

(A)
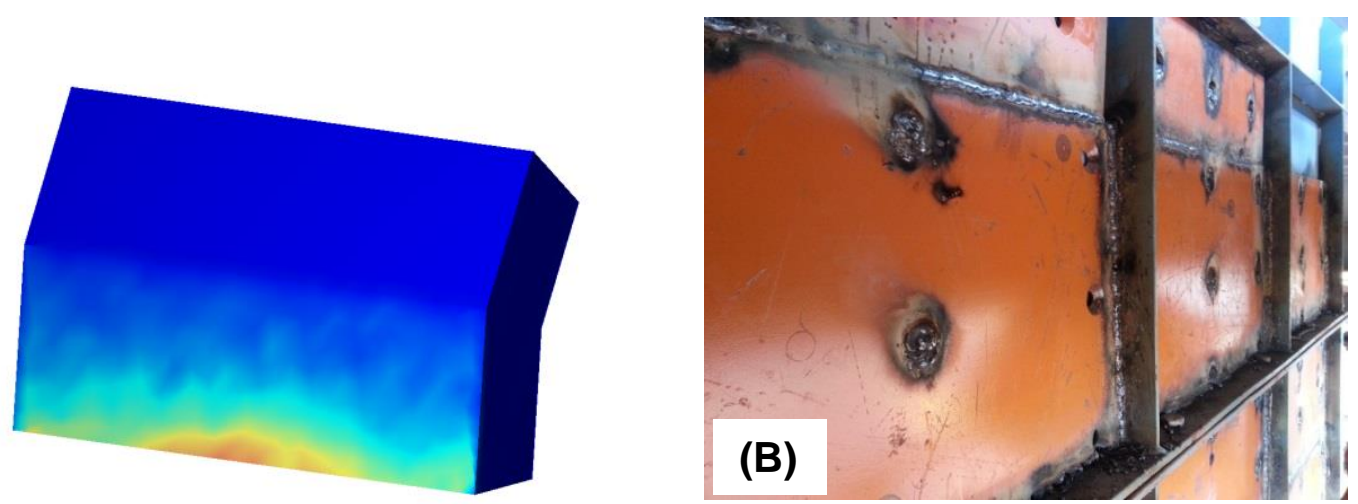

Figura 5: (A) Resultado do Impacto no modulo de transição. (B) Instalação de placas de desgaste devido a furos na chapa do modulo de transição do chute de by-pass. 
O impacto do minério com o rolo móvel da prensa foi avaliado e foi constatado que o impacto gerado neste componente pode gerar desgaste prematuro dos pinos do rolo. Este desgaste prematuro contribui para o desbalanceamento e consequentemente falhas em componentes auxiliares do rolo móvel. Verificou-se no local que estava havendo impacto do material nos rolos, conforme previsto pela simulação. Um anteparo foi desenvolvido para a proteção do rolo com chapas de desgaste. A Figura 6 (A) e (B) apresentam a simulação e a alternativa adotada para evitar o desgaste dos rolos.

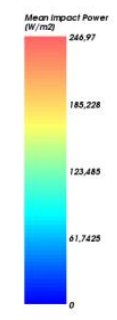

(A)
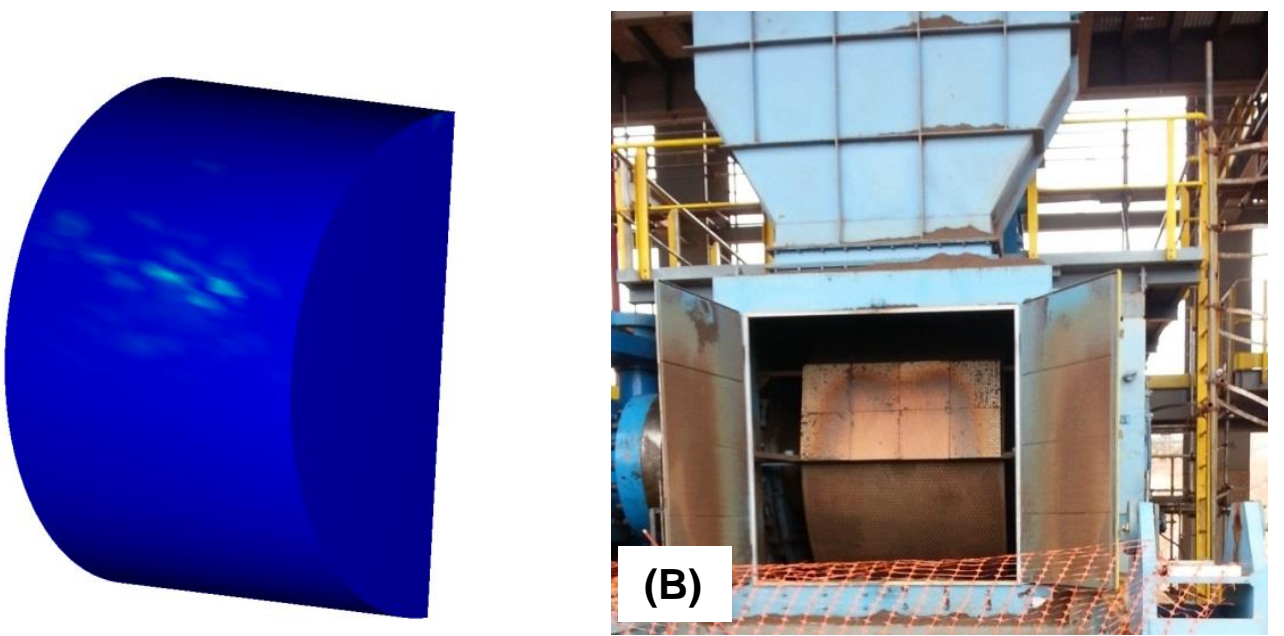

Figura 6: (A) Resultado do Impacto no rolo móvel. (B) Anteparo removível instalado para a proteção do rolo móvel.

Verificou-se através de um volume de controle a possibilidade de entupimento na estrutura vertical onde estão localizadas as caixas de rochas. A Figura 7 apresenta o acumulo de material com o tempo.

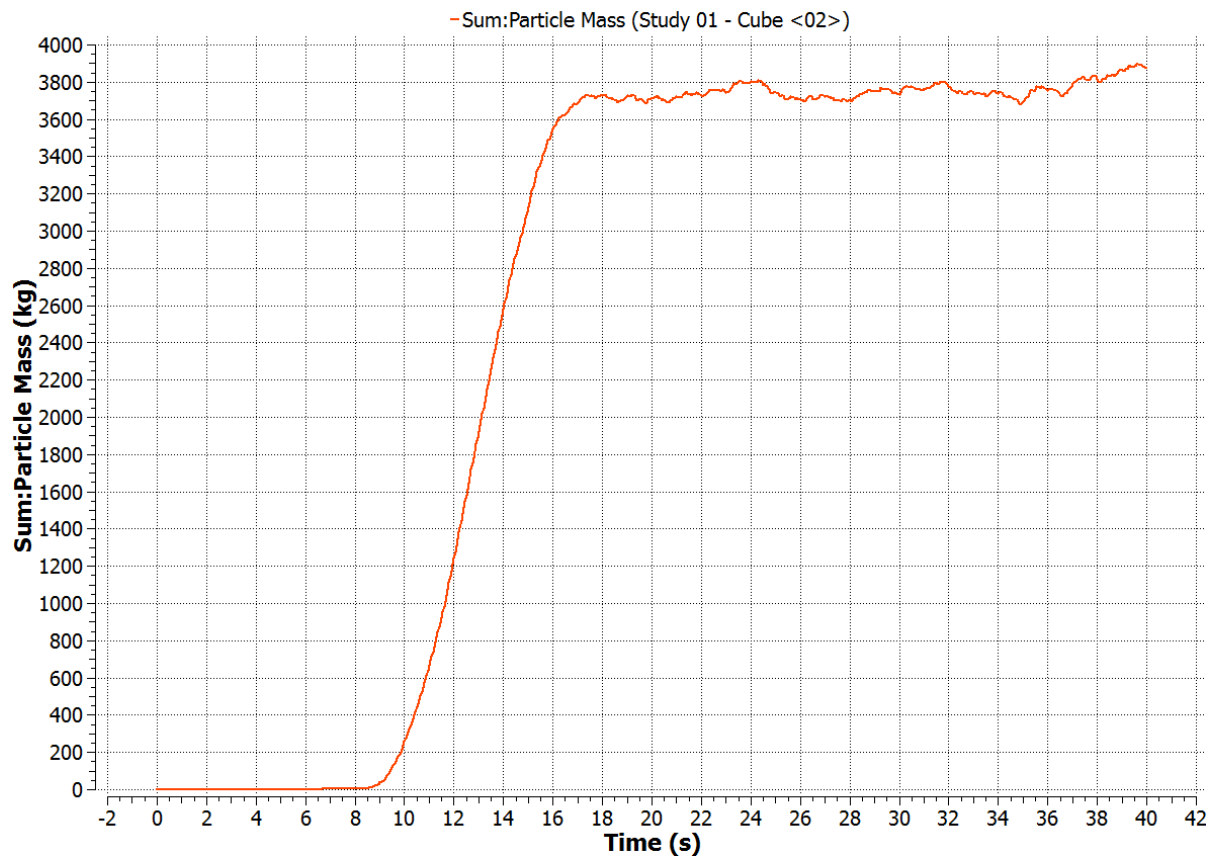

Figura 7: Acumulo de material versus tempo. 
Pode-se observar que a massa dentro do volume de controle se estabiliza em $18 \mathrm{~s}$ e sofre pequenas alterações daí em diante. Através desta análise pode-se atestar que não há obstrução de fluxo nesta região.

A Figura 8 mostra a eficiência de redução da velocidade das partículas com a disposição das caixas de rochas.
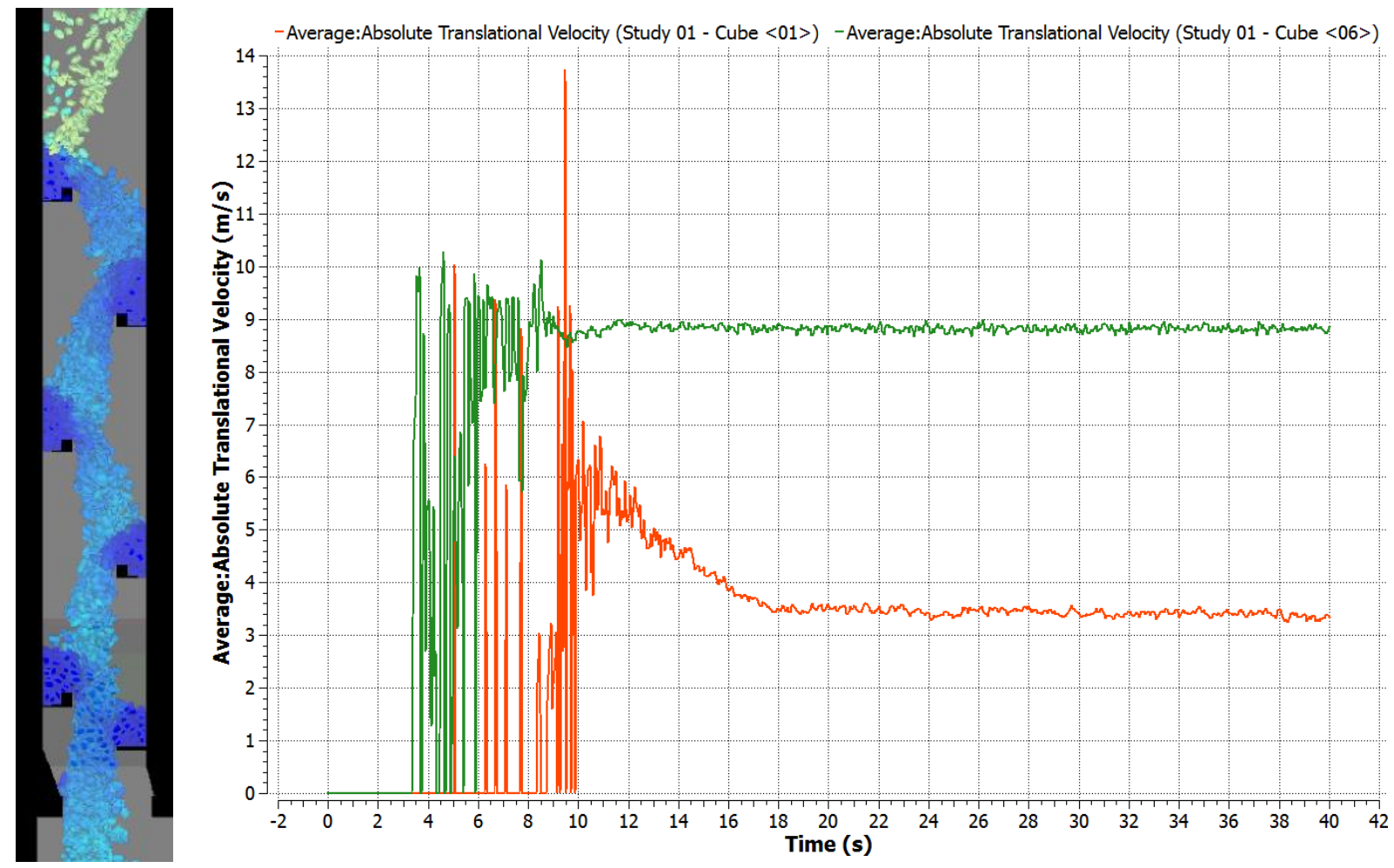

Figura 8: Comparação entre as velocidades médias das partículas na entrada e na saída da estrutura vertical em relação ao tempo.

De acordo com os resultados houve uma redução significativa da velocidade média das partículas de $142 \%$, demonstrando a eficiência das caixas de rocha. Os valores em verde demonstram a média da velocidade no início das caixas de rocha e os valores em vermelho representam a média no final das caixas de rochas.

\section{CONCLUSÕES}

As simulações indicaram a necessidade de proteção em determinadas áreas do chute estudado para evitar o desgaste prematuro. Estas indicações foram constatadas em campo. Não foi verificado na região das caixas de rocha obstrução de fluxo, observou-se uma estabilização da massa dentro do volume de controle. As caixas de rocha se mostraram eficientes na redução da velocidade das partículas. 


\section{AGRADECIMENTOS}

Agradecemos a Anglo American pela autorização para a divulgação dos dados apresentados neste trabalho além de todos os profissionais envolvidos neste estudo.

\section{REFERÊNCIAS BIBLIOGRÁFICAS}

1. Cundall, P., and Strack, O.D.L. A discrete numerical model for granular assemblies, Géotechnique, 1979, (29): 47-65.

2. Nasato, D. S. Desenvolvimento de Acoplamento Numérico entre o Método dos Elementos Discretos (DEM) e o Método dos Elementos Finitos. [Dissetação de Mestrado]. Faculdade de Engenharia Química, UNICAMP, Campinas, São Paulo; 2011. 